\title{
Preparation of Viscose Fibres Stripped of Reactive Dyes and Wrinkle- Free Crosslinked Cotton Textile Finish
}

\author{
Helena Wedin ${ }^{1}$. Ellinor $\mathrm{Niit}^{2} \cdot$ Zaheer Ahmad Mansoor $^{3}$. Anna Rúna Kristinsdottir ${ }^{4}$ Hanna de la Motte ${ }^{5}$. \\ Christina Jönsson $^{4} \cdot$ Åsa Östlund ${ }^{1} \cdot$ Christofer Lindgren ${ }^{3}$
}

Published online: 5 May 2018

(c) The Author(s) 2018

\begin{abstract}
The chemical recycling of cellulosic fibres may represent a next-generation fibre-fibre recycling system for cotton textiles, though remaining challenges include how to accommodate fibre blends, dyes, wrinkle-free finishes, and other impurities from finishing. These challenges may disrupt the regeneration process steps and reduce the fibre quality. This study examines the impact on regenerated viscose fibre properties of a novel alkaline/acid bleaching sequence to strip reactive dyes and dimethyloldihydroxyethyleneureas (DMDHEU) wrinkle-free finish from cotton textiles. Potentially, such a bleaching sequence could advantageously be integrated into the viscose process, reducing the costs and environmental impact of the product. The study investigates the spinning performance and mechanical properties (e.g., tenacity and elongation) of the regenerated viscose fibres. The alkaline/acid bleaching sequence was found to strip the reactive dye and DMDHEU wrinkle-free finish from the cotton fabric, so the resulting pulp could successfully be spun into viscose fibres, though the mechanical properties of these fibres were worse than those of commercial viscose fibres. This study finds that reactive dyes and DMDHEU wrinkle-free finish affect the viscose dope quality and the regeneration performance. The results might lead to progress in overcoming quality challenges in cellulosic chemical recycling.
\end{abstract}

Keywords Decolourization $\cdot$ Chemical textile recycling $\cdot$ Crosslinking agent $\cdot$ Easy-care $\cdot$ Reactive dye

\section{Background}

As the global demand for textile fibres is expected to increase, based on the estimated global increase in population and increased standards of living, there will be an estimated cellulosic gap of five million metric tonnes of fibres

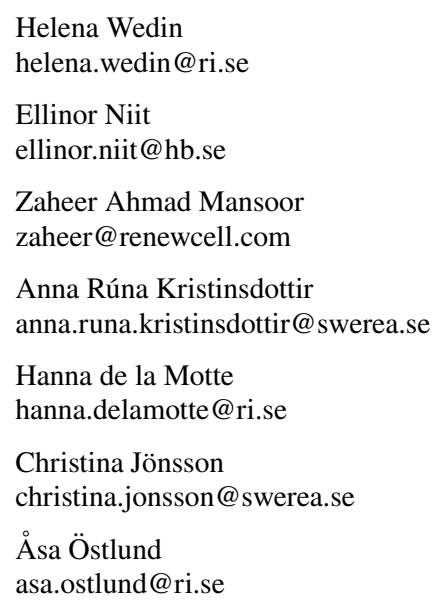

globally by 2020 [1]. This expected shortfall in cellulosebased fibres may be somewhat reduced by using post-production and post-consumer cotton as raw material in regenerated man-made cellulosics, such as viscose or lyocell.

Recycling textiles such as cotton into regenerated fibres has environmental benefits compared with incineration with

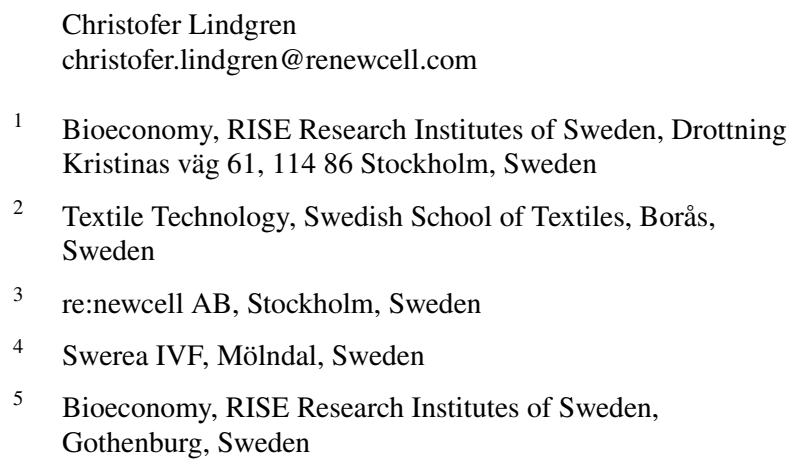


energy recovery in the Nordic region, and the environmental performance of the secondary raw material is expected to be similar to that of other regenerated cellulosic fibres [2]. However, the fibre-to-fibre recycling route is still challenging for both synthetic and natural fibres, and to achieve feasible business models using reclaimed fibres as the raw material, it is crucial that the fibres be of virgin quality. Notably, the use phase of the garment can affect the fibres at a molecular level and reduce the degree of polymerization of the cellulose polymer [3], limiting the options for recycling. One method to recycle cotton could be to regenerate the cotton cellulose into man-made cellulosic fibres via chemical recycling. A potential benefit of chemically recycling cotton into man-made fibres, in contrast to mechanical recycling (common for cotton today), is that, besides obtaining quality similar to that of virgin regenerated fibres, impurities could be removed and other fibres separated in parallel. This is important because impurities such as additives, colourants, and other finishing agents in cotton fabric may represent major technical problems in maintaining the continuity of chemical recycling, and may also transfer potentially hazardous chemicals present in the raw material. Increasing recycling that contributes to a toxin-free environment entails addressing potential risks in an integrated and effective way. To meet the requirements of the regeneration process, the impurity content of textile pulp needs to be at the same level as that of commercial dissolving pulps. It is therefore essential to reduce the impurity content by decolourizing dyed cotton before dissolving.

Typical colourants encountered during decolourization are reactive dyes, since these are currently the most used in dyeing cotton textiles. The success of reactive dyes is attributable to their shade versatility, application flexibility, compatibility with cotton, and stability in laundering conditions. Besides dyes, a type of finishing agent widely used in the textile industry is wrinkle-free finish, which imparts wrinkle resistance to cellulosic materials such as cotton. The modified dimethyloldihydroxyethyleneureas (DMDHEUs) are, commercially, the most widely used today because they can be categorized as low-formaldehyde reactants [4] and require that the curing temperature exceed $130{ }^{\circ} \mathrm{C}$ [5]. The latter is beneficial because the treated fabrics can remain unaffected for a long time before after curing, making them easier to handle in textile finishing. Haule et al. [6] demonstrated that a typical DMDHEU wrinkle-free (i.e., easy-care) finish applied to cotton is durable after multiple launderings. Hence, post-consumer cotton waste could well contain wrinkle-free finish residues. This durability during laundering is because the DMDHEU finish is covalently bonded to the cellulose molecule.

A common approach to removing contaminants bonded to cellulosic substrates, such as wood pulp or cotton textile, is bleaching. Bleaching of cellulosics to obtain whiter and brighter material is commonly performed using various chemical processes, or sequences of these, involving, for example, sodium hydroxide, dithionite, alkaline hydrogen peroxide, ozone, and hypochlorite treatments. The removal of impurities from secondary cotton raw materials has been investigated by several groups [6-10]. To remove DMDHEU in particular, alkaline conditions have been demonstrated to work efficiently [11, 12]. Furthermore, Haule et al. [6] demonstrated that the easy-care DMDHEU crosslinker could successfully be removed from cotton cellulose by applying $23 \mathrm{wt} \%$ sodium hydroxide solution at $100{ }^{\circ} \mathrm{C}$, followed by concentrated sulphuric acid and then rinsing in $23 \mathrm{wt} \%$ sodium hydroxide solution at $100{ }^{\circ} \mathrm{C}$. A later publication reported that cotton pulp was successfully dissolved and spun into regenerated lyocell fibres with mechanical properties superior to those of standard lyocell fibres [13]. Likewise, reactive dyes are covalently bonded to the cellulose polymer and need to be removed in a pretreatment stage.

The present study investigated the stripping of DMDHEU finish and reactive dyes using a novel sequential alkaline/ acid pretreatment bleaching process followed by the evaluation of subsequent viscose fibre regeneration performance. The alkaline part of the bleaching sequence was performed to strip the cotton of dyes and finish, whereas the acid part of the sequence decreased the degree of polymerization to a suitable level for the dissolution window of viscose regeneration. From a sustainability perspective, the selected bleaching sequence could be integrated into the viscose process, leading to reduced costs and environmental impacts. Stripping has previously been performed on wrinkle-free treated fabrics, but not on fabrics with both anti-wrinkle treatment and dye. The resulting viscose fibres were tested for technical performance, i.e., mechanical properties such as tenacity and elongation at break, as indicative of spinning results.

\section{Materials and Methods}

\section{Materials}

Unbleached greige plain woven cotton fabric sheets were generously supplied by Senstex, Sweden. The fabric had a yarn count of Ne 14/12 (warp/weft), thread count (threads/ inch) of 60/56 (warp/weft), and grammage per square metre of 225. Greige fabric comes directly from the loom and contains natural colour and impurities (i.e., extractives).

\section{Chemicals}

The reactive dyes Levafix Yellow CA, Levafix Red CA, and Levafix Blue CA and the auxiliaries Sera Gal C-RFX and Sera Fast C-RD were all obtained from DyStar Group (Singapore). Sera Gal C-RFX is a sulphosuccinate surfactant 
and Sera Fast C-RD a carboxylate surfactant. The Levafix dyes have an Oeko-Tex Passport certificate, i.e., were developed to meet the requirements of Oeko-Tex standard 100 concerning health and environmental performance. Bifunctional dyes have a higher degree of fixation due to two reactive groups that bind covalently [14]. Levafix Yellow CA is a monoazo reactive dye with two reactive groups (fluorotriazine and sulphatoethylsulphone), Levafix Red $\mathrm{CA}$ is a hydrogen-acid based monoazo reactive dye with two reactive groups (fluorotriazine and difluoropyrimidine), and Levafix Blue CA is a copper formazan dye (containing complexed copper) with two reactive groups (fluorotriazine and sulphatoethylsulphone). The other chemicals were of technical grade. The acetic acid $(60 \mathrm{wt} \%)$ and sodium hydroxide ( $45 \mathrm{wt} \%$ ) were supplied by Brenntag, Sweden and the sodium chloride (industrial salt) was supplied by Akzo Nobel Chemicals, the Netherlands.

Stabitex ETR Plus, a wrinkle-free crosslinking agent, was obtained from Pulcra Chemicals (Geretsried, Germany). It consists of $N, N^{\prime}$-dimethylol-4,5-dihydroxyethyleneurea (DMDHEU) and has an inbuilt catalyst. Stabitex ETR Plus releases a minimal amount of formaldehyde, and was also developed for textiles that conform to the Oeko-Tex

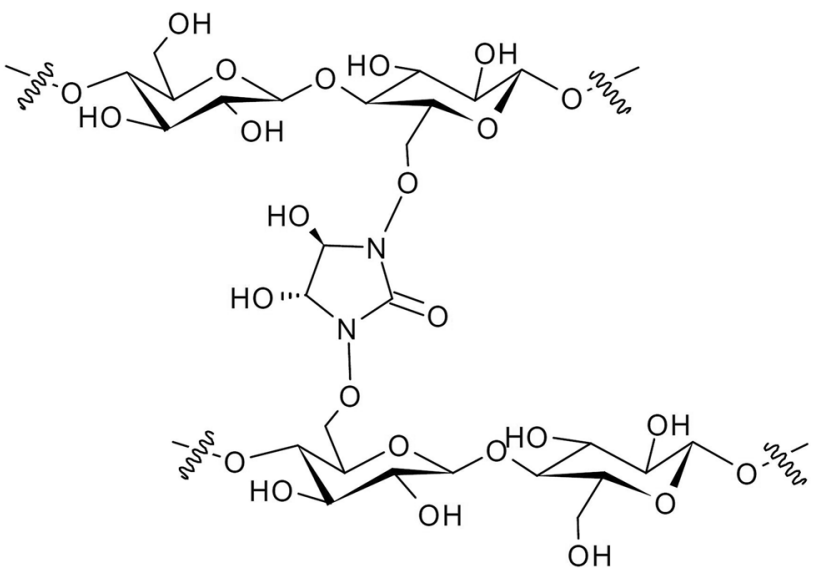

Fig. 1 Wrinkle-free finish (centre) crosslinking with two cellulose chains (top and bottom)
Standard 100. Figure 1 shows the structure of the DMDHEU crosslinked to cellulose.

\section{Application of Reactive Dyes and DMDHEU Wrinkle-Free Finish to Cotton}

The reactive dyes and DMDHEU wrinkle-free finish were applied to cotton in the steps described in Table 1; four different samples were produced: (1) dyed, (2) combined dyed and wrinkle free, (3) wrinkle free, and (4) untreated.

The fabrics were washed before dyeing in a washing machine at $90{ }^{\circ} \mathrm{C}$ for 15 min with a charge of $1 \mathrm{~g} / \mathrm{L}$ of SeraGal C-RFX to desize the water-soluble carboxymethyl cellulose. A combination of Levafix yellow, red, and blue was charged to obtain a grey medium-depth colour in the fabric. Using a trichrome (i.e., three dyestuffs) combination in one step is a common way to achieve the desired shade. The Levafix dyestuffs were developed and optimized for medium-depth shades. The concentration of the Levafix Yellow CA was $0.14 \%$, Levafix Red CA $0.08 \%$, and Levafix Blue CA $0.2 \%$. The dyeing was performed at Korallen in Kinnarumma, Sweden in a rebuilt industrial washing machine. Before dye addition, $25 \mathrm{~g} / \mathrm{L}$ of sodium chloride and $1 \mathrm{~g} / \mathrm{L}$ of Sera Gal C-RFX were charged. After $10 \mathrm{~min}$, the dyes were added and $0.36 \mathrm{~g} / \mathrm{L}$ of sodium hydroxide solution was pumped continuously for $60 \mathrm{~min}(0.24 \mathrm{~g} / \mathrm{min})$. The dyeing and three subsequent rinsing steps were performed at $60{ }^{\circ} \mathrm{C}$ for $3 \mathrm{~h}$. In the second rinsing, $0.6 \mathrm{~g} / \mathrm{L}$ of acetic acid was charged and in the third rinsing $1 \mathrm{~g} / \mathrm{L}$ of SeraFast C-RD was charged. The drying was performed at $60^{\circ} \mathrm{C}$ for $30 \mathrm{~min}$ in a tumble dryer. To minimize the effects of the different process steps, the untreated and wrinkle-free treated fabrics were processed in the same equipment without dye addition, but with SeraGal C-RFX and SeraFast C-RD auxiliaries.

To apply wrinkle-free finish to cotton, the cotton fabric was subsequently treated with $40 \mathrm{~g} / \mathrm{L}$ of DMDHEU (Stabitex ETR Plus) at pH 5.5 (adjusted with acetic acid) in a washing machine for $20 \mathrm{~min}$ at $40^{\circ} \mathrm{C}$. The tumble drying was performed at $85^{\circ} \mathrm{C}$ for $30 \mathrm{~min}$. The manufacturer recommends drying the agent at $110-120{ }^{\circ} \mathrm{C}$ with subsequent curing for $4 \mathrm{~min}$ at $150^{\circ} \mathrm{C}$ (or shock curing for $30-60 \mathrm{~s}$ at $180-170{ }^{\circ} \mathrm{C}$ ).
Table 1 Experimental outline of the fabric treatments generating the four samples

\begin{tabular}{lllll}
\hline & $\begin{array}{l}\text { Dyed and } \\
\text { wrinkle } \\
\text { free }\end{array}$ & Wrinkle free & Dyed Untreated \\
\hline Pre-washing at $90{ }^{\circ} \mathrm{C}$ & Yes & & & \\
Dyeing at $60{ }^{\circ} \mathrm{C}+$ drying at $60^{\circ} \mathrm{C}$ & Yes $^{\mathrm{a}}$ & No (with auxiliaries) & Yes $^{\mathrm{a}}$ & No (with auxiliaries) \\
Wrinkle-free treatment + drying at $85^{\circ} \mathrm{C}$ & Yes $^{\mathrm{b}}$ & Yes $^{\mathrm{b}}$ & No & No \\
Curing at $160{ }^{\circ} \mathrm{C}$ for 3 min & Yes & & & \\
\hline
\end{tabular}

${ }^{\text {a}}$ Reactive dye (Levafix CA 3-colour combination) and auxiliaries

${ }^{\mathrm{b}}$ Stabitex ETR Plus, $40 \mathrm{~g} / \mathrm{L}$ 
To compensate for the lower drying temperature in the tumble drying, the curing was performed at $160{ }^{\circ} \mathrm{C}$ for $3 \mathrm{~min}$ in a hot press to ensure that crosslinking was taking place. It is common to use softener after the treatment, but to elucidate only the effect of the crosslinking agent, softener was not used in this study. The acid wash at $\mathrm{pH} 5.5$ and subsequent drying were not performed on the untreated and the dyed fabrics, since they were assumed to have a negligible effect on the properties of the cellulose polymer.

Subsequent washing to remove any unreacted DMDHEU was performed three times in a $0.01 \mathrm{M}$ sodium hydroxide solution ( $\mathrm{pH} \mathrm{12}$ ) for $1 \mathrm{~h}$ at $60{ }^{\circ} \mathrm{C}$ followed by three times in a $0.01 \mathrm{M} \mathrm{HCl}$ solution $(\mathrm{pH} 3)$ for $1 \mathrm{~h}$ at $60^{\circ} \mathrm{C}$.

\section{Bleaching}

Bleaching, or stripping, was used to decolourize the dyes and hydrolyse the crosslinking agent. The bleaching was performed in a two-step reaction process in which the first step was alkaline and the second acid. The sequence entailed the use of a bleaching agent, but the type of bleaching agent and exact conditions cannot be disclosed for reasons of commercial confidentiality. The degree of polymerization of the cellulose was adjusted to be in the dissolving pulp range for the viscose manufacturing process. The chemical charges in the bleaching were equal for all samples and based on the limiting viscosity number of the reference sample. The reaction time was adjusted to reach the target limiting viscosity number of $500-600 \mathrm{~mL} / \mathrm{g}$ for all pulps.

\section{Viscose preparation and spinning}

The preparation of viscose solution comprises several steps, in the following order: steeping, pressing, shredding, aging, xanthation, dissolving, and ripening. The steeping was carried out for $60 \mathrm{~min}$ at $40{ }^{\circ} \mathrm{C}$ with $18 \mathrm{wt} \%$ sodium hydroxide. In the pressing step, a pressing factor of 3.0 was used. The shredding was performed at $50{ }^{\circ} \mathrm{C}$ for $45 \mathrm{~min}$ followed by aging at $50{ }^{\circ} \mathrm{C}$. A pre-aging curve was calculated to obtain the optimal time to reach an equal limiting viscosity number before xanthation, since a controlled limiting viscosity number after aging is required for good processability $[15,16]$. The optimal aging time was $145 \mathrm{~min}$ for the wrinkle-free treated and untreated samples and $160 \mathrm{~min}$ for the dyed and combined dyed and wrinkle-free treated samples. Xanthation was performed for $2.5 \mathrm{~h}$ at $32{ }^{\circ} \mathrm{C}$ with the addition of $36 \mathrm{wt} \%$ carbon disulphide; the cellulose xanthate thus formed was subsequently dissolved for $3 \mathrm{~h}$ at $7{ }^{\circ} \mathrm{C}$. Finally, the viscose solution was ripened for $16 \mathrm{~h}$ at room temperature. Before wet spinning the viscose solution, the solution was filtered and deaerated. The wet spinning was performed at $50{ }^{\circ} \mathrm{C}$ using a spin-bath composition of $130 \mathrm{~g} / \mathrm{L}$ of sulphuric acid, $310 \mathrm{~g} / \mathrm{L}$ of sodium sulphate, and $9.5 \mathrm{~g} / \mathrm{L}$ of zinc sulphate.
Godet speed 1 was set to $18.8 \mathrm{~m} / \mathrm{min}$, giving a draw ratio of 1.7 , and godet speed 2 was set to $29.9 \mathrm{~m} / \mathrm{min}$.

\section{Characterization}

Several methods have been used to properly characterize the viscose solution for spinnability, chemical residues in the pulp, and performance of the generated fibre. Elemental analysis to detect the impurities was performed according to ISO 16948 using a CHN 628 carbon/hydrogen/nitrogen determinator from Leco (St. Joseph, MI, USA). The error associated with the measurement is $\pm 0.02 \%$.

The limiting viscosity number was determined in cupriethylenediamine (CED) solution using a capillary viscometer following the ISO 5351:2010 standard method. Solubility (s) in the CED solution was determined according to Haule et al. [6], with the modification that the filtration was conducted in two steps, first through a sintered P1 or P3 glass filter and then through a GF/A glass filter (Whatman, Maidstone, UK). The two wrinkle-free-finish samples (one dyed and one undyed) were filtered through P1 followed by GF/A filters, the dyed through P3 followed by GF/A filters, and the untreated sample through only the GF/A filter. When s was $100 \%$ the sample was considered $100 \%$ soluble and was tested for the limiting viscosity number.

The ISO brightness (D65), used to monitor the dye removal from fabrics and pulp during bleaching, was determined according to ISO 2470:1999.

Fourier transform infrared (FTIR) spectroscopy spectra were recorded on a Frontier FTIR device (Perkin-Elmer, Waltham, MA, USA) to investigate the occurrence of the reacted dye and wrinkle-free finish on the treated cotton fabrics. The FTIR was equipped with a Frontier UATR accessory ( $\mathrm{ZnSe}$ with reflection top-plate and pressure arm). Spectrum 10 software was used with 10 and 128 scans per sample; the spectra were normalized at $2900 \mathrm{~cm}^{-1}(\mathrm{C}-\mathrm{O}-\mathrm{C}$ stretching vibration).

The viscose solution was characterized in terms of the cellulose content, alkaline content, ball fall, gamma number, and reduced filter-clogging number $\left(K_{r}\right)$. The cellulose content of the viscose was determined by pressing the viscose solution between two glass plates. The plates were separated by sliding and then placing them in a sulphuric acid bath until the viscose film had loosened from the plates. The cellulose film was washed thoroughly with water and dried by removing excessive moisture with a filter cloth. The film was then placed in an oven at $105^{\circ} \mathrm{C}$ for $4 \mathrm{~h}$. The cellulose content was calculated as the weight of the sample after drying divided by the weight of the viscose solution.

The alkali content of the viscose was determined by first weighing about $2.5 \mathrm{~g}$ of viscose and then dissolving it in $100 \mathrm{~mL}$ of deionized water with a magnetic stirrer. The solution was boiled and $10 \mathrm{~mL}$ of $0.5 \mathrm{~mol} / \mathrm{dm}^{3}$ sulphuric 
acid was added. When the solution reached room temperature, several drops of phenolphthalein were added and then the solution was titrated with $1.0 \mathrm{~mol} / \mathrm{dm}^{3}$ sodium hydroxide until the solution changed colour. The following equation was used to calculate the alkali content: the viscose solution. A lower value means faster coagulation, making the material less suited for spinning.

All fibres were tested for tenacity and elongation in both the conditioned and wet states. A Vibroskop 400 instrument (Lenzing Instruments, Gampern, Austria) was used

Alkali content $(\%)=\frac{\left(V_{\mathrm{H}_{2} \mathrm{SO}_{4}} \times 2 \times c_{\mathrm{H}_{2} \mathrm{SO}_{4}}-V_{\mathrm{NaOH}} \times c_{\mathrm{NaOH}}\right) \times 40 \times 100}{m \times 1000}$

where $V$ is volume in $\mathrm{mL}, c$ is concentration in $\mathrm{mol} / \mathrm{dm}^{3}$, and $m$ is mass of the viscose solution in grams.

The gamma number is defined as the number of xanthogenate groups per 100 anhydroglucose units and describes the degree of substitution, with the highest gamma number being 300. A viscose sample of about $1 \mathrm{~g}$ was dissolved in $50 \mathrm{~mL}$ of $1 \mathrm{wt} \% \mathrm{NaOH}$ by stirring for $30 \mathrm{~min}$. The solution was then transferred into a $100-\mathrm{mL}$ volumetric flask and diluted with $1 \mathrm{wt} \% \mathrm{NaOH}$. The flask was shaken and $5 \mathrm{~mL}$ of solution were transferred to a bottle containing $1.8 \mathrm{~g}$ of Amberlite IRA 402 ion-exchange resin (Sigma-Aldrich, St. Louis, MO, USA). The solution was left to react with the resin for $10 \mathrm{~min}$, after which it was filtered using Munktell 5 filter paper (Fisher Scientific, Waltham, MA, USA) into a $250-\mathrm{mL}$ volumetric flask and diluted with deionized water. Measurements were made with a CARY 100 Scan UV/vis spectrophotometer (Agilent, Santa Clara, CA, USA) at a wavelength of $303 \mathrm{~nm}$. The gamma number was calculated according to the following equation:

Gamma number $=\frac{A b s_{303 \mathrm{~nm}} \times 546.48}{m_{\text {viscose }} \times c_{\text {cellulose }}}$

where $A b s$ is absorbance at $303 \mathrm{~nm}, m$ is mass of the viscose solution in grams, and $c$ is cellulose concentration in $\%$.

The reduced filter-clogging number $\left(K_{r}\right)$ was determined according to Treiber [15], Treiber et al. [17], and the ZELLCHEMING III/6B/68 standard method [18]. $K_{r}$ is obtained by filtering the viscose solution and measuring the filtered amount and ball fall time. The filtered amount is measured after $20 \mathrm{~min}$ and again after $1 \mathrm{~h}$. The falling ball time was measured using a $1 / 8$-inch steel ball allowed to fall in a 200-mm-deep column filled with the viscose sample solution.

$K_{r}=\frac{10^{4} \times 2 \times\left(\left(\frac{t_{2}}{M_{2}}\right)-\left(\frac{t_{1}}{M_{1}}\right)\right)}{\left(t_{2}-t_{1}\right) \times \eta^{0.4}}$

where $t_{l}=1200 \mathrm{~s}(20 \mathrm{~min}), t_{2}=3600 \mathrm{~s}(60 \mathrm{~min}), M_{1}=$ total amount of viscose in the beaker after $20 \mathrm{~min}, M_{2}=$ total amount of viscose in the beaker after $60 \mathrm{~min}$, and $\eta=$ ball falling time in seconds.

The ripening index before spinning indicates how fast the spin dope coagulates and is a measure of the quality of according to SS-EN ISO 5079 to determine the fibre titre (i.e., linear density of the fibres), which is derived from the vibration frequency of the fibre. A Vibrodyn 400 instrument (Lenzing Instruments) was used according to SS-EN ISO 5079, ISO1130-1975, and ISO1973 to determine the fibre elongation (i.e., extension at break) and tenacity. Twenty fibres from each sample were tested. The standard deviation of the mechanical properties was adjusted for a $95 \%$ confidence interval.

\section{Results and Discussion}

\section{Dyeing and Wrinkle-Free Treatment}

Unbleached greige cotton fabric was dyed and wrinkle-free treated, individually and in combination. As a reference, an untreated sample undergoing the same process steps was used, but without the addition of dyes or wrinkle-free agent. The treatments are outlined in Table 1. Four sample types were produced: combined dyed and wrinkle free, dyed, wrinkle free, and untreated.

Elemental analysis of nitrogen in the original greige cotton fabric revealed a nitrogen content of $0.18 \%$, probably originating from the impurities in the untreated fabric. Table 2 shows the nitrogen content of the untreated and treated cotton fabric after curing. The untreated cotton fabric contained $0.11 \%$ nitrogen, whereas the wrinkle-free DMDHEU-treated fabrics had a slightly higher nitrogen

Table 2 Elemental nitrogen composition of cotton treated with reactive dyes and DMDHEU wrinkle-free finish (i.e., before bleaching) and after subsequent bleaching

\begin{tabular}{lll}
\hline Sample & \multicolumn{2}{l}{ Nitrogen content, abs \% atomic } \\
\cline { 2 - 3 } & $\begin{array}{l}\text { Before bleaching } \\
\text { (after curing) }\end{array}$ & $\begin{array}{l}\text { After } \\
\text { subsequent } \\
\text { bleaching }\end{array}$ \\
\hline Dyed and wrinkle free & $0.19 \pm 0.02$ & $0.04 \pm 0.02$ \\
Wrinkle free & $0.19 \pm 0.02$ & $0.04 \pm 0.02$ \\
Dyed & $0.14 \pm 0.02$ & $0.03 \pm 0.02$ \\
Untreated & $0.11 \pm 0.02$ & $0.02 \pm 0.02$ \\
\hline
\end{tabular}




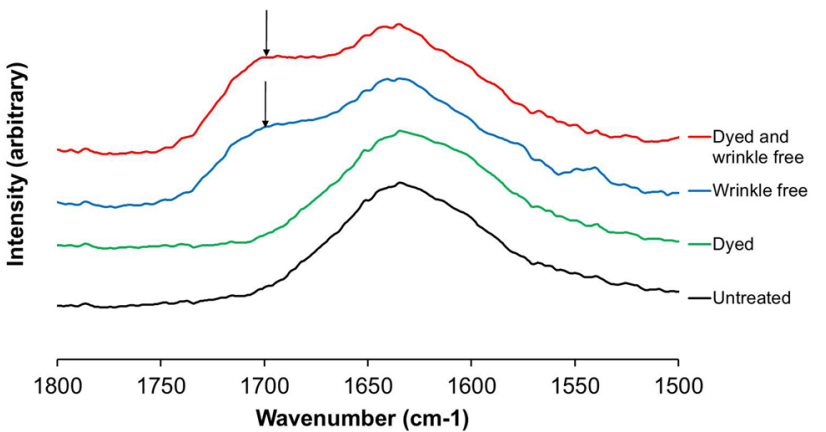

Fig. 2 FTIR spectra of cotton fabrics finished with reactive Levafix dyes and wrinkle-free DMDHEU finish. The spectra are presented separately content of $0.19 \%$, indicating the presence of DMDHEU. However, this nitrogen content indicated only the presence of the DMDHEU agent, but not whether the crosslinking reaction had taken place. To confirm this reaction, FTIR spectra were analysed before and after sequential treatment with sodium hydroxide and hydrochloric solution to remove any unreacted DMDHEU. The peak at $1700 \mathrm{~cm}^{-1}$ is associated with the carbonyl stretch of DMDHEU, whereas the peak at $1700 \mathrm{~cm}^{-1}$ after the alkaline and acid washing confirmed that the crosslinking reaction had taken place (Fig. 2).
Fig. 3 Dyed and DMDHEU wrinkle-free-treated cotton fabrics before bleaching and the corresponding pulps and viscose fibres after bleaching

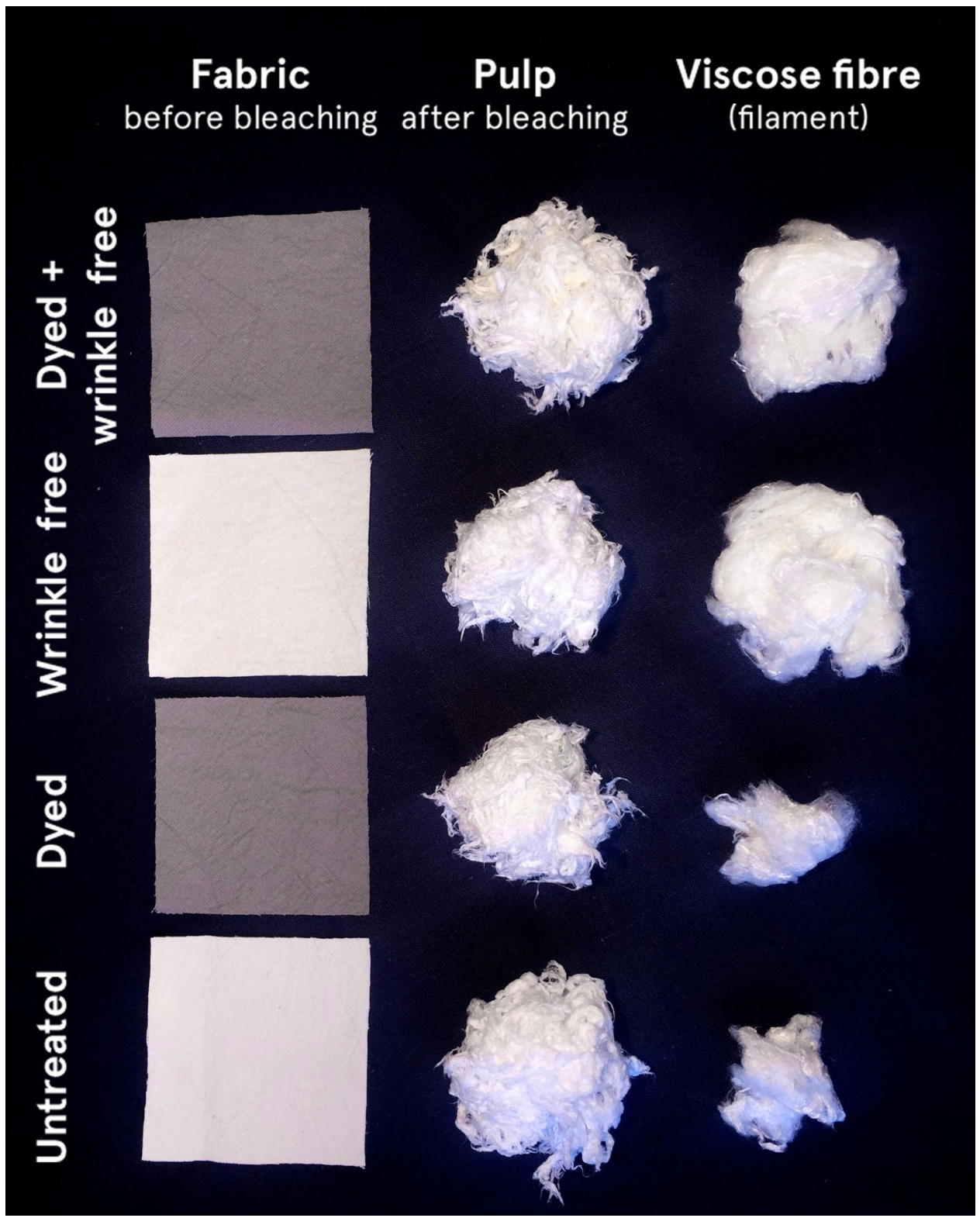




\section{Bleaching of Cotton Fabrics}

A sequential alkali/acid bleaching step was used to decolourize the dyes and hydrolyse the crosslinking agent. Figure 3 depicts the samples before and after bleaching.

As shown in Table 2, elemental analysis of the nitrogen content after the alkaline/acid bleaching indicated effective hydrolysis of the wrinkle-free finish to almost the same level, i.e., $0.04 \% \pm 0.02$, as in the untreated cotton fabric, i.e., $0.02 \%$. Furthermore, the FTIR spectra after bleaching (Fig. 4) confirmed the elemental analysis data, showing a decrease in the band at $1700 \mathrm{~cm}^{-1}$ to the same level as in the untreated fibres. The effectiveness of using a sequential alkaline/acidic step to hydrolyse the wrinkle-free cotton treated with DMDHEU is in line with previous results of Haule et al. [6]. They proposed that the sodium hydroxide treatment partially hydrolysed the $\mathrm{C}-\mathrm{N}$ bond, leaving DMDHEU mono-functionally bound to the cellulose. This explained why the crosslinking effect was absent in their study, while the nitrogen content still did not reach zero. A subsequent sulphuric acid treatment was necessary to obtain full hydrolysis and a nitrogen content of zero. In the present study, the alkaline step involved a bleaching chemical in an alkaline solution followed by treatment with a mildly acidic solution.

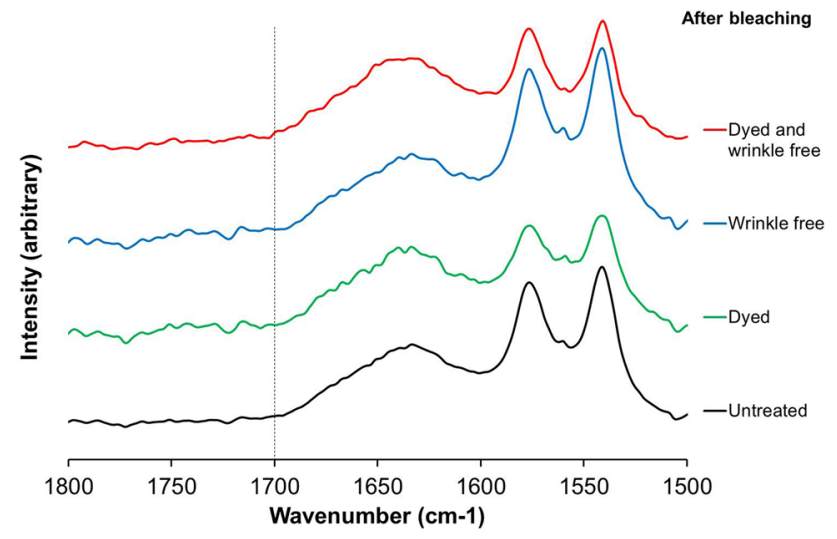

Fig. 4 FTIR spectra of cotton fabrics finished with reactive Levafix dyes and wrinkle-free DMDHEU agent and then bleached with sequential alkali/acid bleaching. The spectra are presented separately
Although the chemicals and conditions are not identical to those used by Haule et al., a decreased nitrogen content is observed here, in line with their results.

Since the structures of the dyes are unavailable, an analysis other than nitrogen content is needed to confirm the removal of the reactive dye. One such method is the ISO brightness measurement, with a high value representing increased brightness. The ISO brightness method is commonly applied to analyse wood dissolving pulp at a wavelength of $457 \mathrm{~nm}$. Table 3 shows the ISO brightness before and after bleaching.

As can be seen in Table 3, the treated samples all displayed lower ISO brightness than did the untreated fabric before bleaching, in the order: untreated $>$ wrinkle free $>$ dyed and wrinkle free $>$ dyed. The bleaching increased the ISO brightness by 30.6 percentage points in the untreated sample from 58.3 to $88.9 \%$, close to commercial dissolving pulp values of 91-92\% ISO brightness. In the treated fabrics, the bleaching corresponded to increases of 29.4, 57.7, and $62.9 \%$ points in ISO brightness for the wrinkle-free, combined dyed and wrinkle-free, and dyed fabrics, respectively. It is clear that the bleaching was effective, because the dyed fabrics had ISO brightness levels of approximately $80-84 \%$ after bleaching, approximately $22-26 \%$ points higher than that of the untreated unbleached cotton fabric, i.e., 58.3\%. It should be noted that this study made no attempt to bleach the dyed fabrics to the same level as the untreated bleached fabric. Logically, doing so would require even higher bleaching charges for the dyed fabrics to obtain similar ISO brightness, so more optimization would be needed to decolourize the fabrics without reducing the target viscosity number. Concerning the ISO brightness method, it is questionable how informative the results are for dyed cotton fabrics, since the method analyses the reflectance at $457 \mathrm{~nm}$ and does not cover the other colours. It is therefore possible that dye residues with or without chromophoric parts could still be bonded to the cellulose after bleaching. Another important concern is the presence of extractive residues in the cotton fibres before dyeing. Usually a fabric is desized, scored, and bleached before dyeing, to remove the sizing agent and extractives that may hinder the strong interaction between the fibre and chemical dyeing agent. The scoring step was
Table 3 Fabric and pulp properties before and after bleaching

\begin{tabular}{|c|c|c|c|c|c|}
\hline \multirow[t]{2}{*}{ Sample } & \multicolumn{2}{|c|}{ ISO brightness (\%) } & \multicolumn{3}{|c|}{ Limiting viscosity number $(\mathrm{mL} / \mathrm{g})$} \\
\hline & $\begin{array}{l}\text { Before } \\
\text { bleaching }\end{array}$ & $\begin{array}{l}\text { After alkaline/ } \\
\text { acid bleaching }\end{array}$ & Before bleaching & $\begin{array}{l}\text { After alkaline } \\
\text { bleaching }\end{array}$ & $\begin{array}{l}\text { After alkaline/ } \\
\text { acid bleaching }\end{array}$ \\
\hline Dyed and wrinkle free & 22.1 & 79.8 & $\mathrm{~N} / \mathrm{A}^{\mathrm{a}}$ & $\mathrm{N} / \mathrm{A}^{\mathrm{a}}$ & 520 \\
\hline Wrinkle free & 57.8 & 87.2 & $\mathrm{~N} / \mathrm{A}^{\mathrm{a}}$ & $\mathrm{N} / \mathrm{A}^{\mathrm{a}}$ & 530 \\
\hline Dyed & 20.9 & 83.8 & $\mathrm{~N} / \mathrm{A}^{\mathrm{a}}$ & $\mathrm{N} / \mathrm{A}^{\mathrm{a}}$ & 540 \\
\hline Untreated & 58.3 & 88.9 & 1700 & 910 & 540 \\
\hline
\end{tabular}

${ }^{a}$ The samples were not $100 \%$ soluble in cupri-ethylenediamine solution 
not undertaken for the greige fabric, however, which might have made it easier to strip the dyes and crosslinker. Despite using an auxiliary suitable for greige fabric, further studies and analyses, preferably on desized, scored, and bleached fabrics, are needed to confirm the decolourization efficiency and presence of residues. However, the present results are indicative of the success of the proposed sequential process.

Table 3 also shows the limiting viscosity numbers before and after bleaching. The limiting viscosity number is a measure estimating the degree of polymerization, with a lower number indicating shorter cellulose polymer chains. The limiting viscosity number is then used as a target value for the chemical addition in the viscose process, to obtain the proper cellulose properties and thus mechanical properties of the fibres. Since the aim was to achieve fibre quality properties corresponding to those of commercial viscose fibres, the limiting viscosity number was set to $500-600 \mathrm{~mL} / \mathrm{g}$, i.e., a common target number for commercial dissolving pulps and thus for "standard" dissolving and spinning conditions. The untreated cotton fabric had an initial limiting viscosity number of $1700 \mathrm{~mL} / \mathrm{g}$. However, only the untreated cotton fabric was soluble in CED solution and could be analysed using the limiting viscosity number method. For the treated fabrics, the cellulose was not completely soluble. The alkaline bleaching step on its own was not effective in dissolving the DMDHEU-treated wrinkle-free cotton in CED, but after the removal of DMDHEU and reactive dyes with the sequential acid step, the pulps were soluble in CED. The low solubility in CED of wrinkle-free DMDHEU-treated cotton fabrics confirms previous findings of Haule et al. [13] that a combined alkaline/acid sequence effectively hydrolyses the DMDHEU wrinkle-free finish. Interestingly, the present study also found that the reactive dyes needed to be removed from the cotton fabric in an alkaline/acid bleaching sequence to obtain the limiting viscosity number of the pulp and hence a material suitable for the viscose process. After alkaline/ acid bleaching, all samples obtained a limiting viscosity number of 520-540 mL/g (Table 3); consequently, "standard" viscose conditions were chosen for the ensuing steps.

\section{Viscose Spinning of the Cotton Pulps}

The bleached pulps were prepared for viscose production and extruded in a spinning bath in a bench-scale unit. The quality of the viscose solutions was characterized by gamma number, cellulose concentration, sodium hydroxide concentration, and ripening index, as shown in Table 4. The gamma number indicates the number of substituted groups of the cellulose polymer, and the ripening index describes how fast the viscose solution coagulates. For a good viscose solution, the numbers should preferably be $30-45$ for the gamma number, $9.5-10.5 \%$ for the cellulose concentration, and $4.0-4.5 \%$ for the sodium hydroxide concentration. A ripening index above nine indicates that the solution has a good coagulation time.

Table 4 shows gamma numbers of $38-42 \%$, cellulose concentrations of $9.2-9.5 \%$, and sodium hydroxide concentrations of 3.8-3.9\%, indicating a high-quality viscose solution. All samples had a ripening index value of 9 or above, and therefore seemed suitable for "standard" commercial pulp conditions in the spinning trials. The stripped reactively dyed and DMDHEU-treated wrinkle-free cotton pulps could be spun into fibres. However, the spinning of the untreated cotton pulp had to be interrupted during the process, due to plugging and accumulated pressure in the spinneret, possibly due to the poor filterability of the viscose solution. Table 4 shows the reduced filter-clogging number, $K_{r}$, which ranged between 976 and 4383 in the order: untreated $>$ dyed $>$ wrinkle-free $>$ dyed and wrinklefree. A high $K_{r}$ number may indicate that the spinning could be negatively affected because the viscose solution contains differing amounts of micro- and macroscopic gel particles. Not only can macro gel particles influence the spinning process, but sub-microscopic particles and aggregates in the filtered spinnable solution can also have an impact [19]. Particles and aggregates, however, are inherent to the viscose dope, contributing to the properties of the dope and ultimately of the fibres. Typical $K_{r}$ values of commercial pulps used in viscose production are 100-1200. Unexpectedly, the combined dyed and wrinklefree treated sample had the lowest $K_{r}$ value of 976 . The dyed and wrinkle-free finished cotton pulps had $K_{r}$ values

Table 4 Viscose solution properties of the four samples

\begin{tabular}{llllllll}
\hline Sample & $\begin{array}{l}\text { Filtration mass } \\
\text { after 20 s/60 s }(\mathrm{g})\end{array}$ & Ball fall (s) & $\begin{array}{l}\text { Gamma } \\
\text { number }\end{array}$ & $\begin{array}{l}\text { Ripening } \\
\text { index }\end{array}$ & $\begin{array}{l}{[\mathrm{NaOH}] \text { in vis- }} \\
\text { cose solution (\%) }\end{array}$ & $\begin{array}{l}\text { [Cellulose] in vis- } \\
\text { cose solution }(\%)\end{array}$ & $\begin{array}{l}\text { Reduced filter- } \\
\text { clogging number, } \\
K_{r}\end{array}$ \\
\hline Dyed and wrinkle free & $3.724 / 3.918$ & 58.54 & 39 & 12 & 3.8 & 9.2 & 976 \\
Wrinkle free & $3.716 / 3.836$ & 35.85 & 42 & 15 & 3.9 & 9.2 & 1285 \\
Dyed & $1.428 / 1.490$ & 71.84 & 38 & 9 & 3.9 & 9.4 & 2376 \\
Untreated & $0.756 / 0.820$ & 65.57 & 40 & 11 & 3.9 & 9.5 & 4383 \\
\hline
\end{tabular}


Table 5 Mechanical properties of spun viscose fibres

\begin{tabular}{|c|c|c|c|c|c|c|}
\hline Sample & Dry $\operatorname{titer}^{1}(\mathrm{dtex})$ & Dry tenacity $^{\mathrm{a}}(\mathrm{cN} / \mathrm{tex})$ & Dry elongation $^{\mathrm{a}}(\%)$ & Wet titer (dtex) & $\begin{array}{l}\text { Wet } \\
\text { tenacity }^{\mathrm{a}} \\
(\mathrm{cN} / \text { tex })\end{array}$ & Wet elongation $^{\mathrm{a}}(\%)$ \\
\hline Dyed and wrinkle free & $2.7 \pm 0.1$ & $13.8 \pm 0.9$ & $15.8 \pm 1.3$ & $2.4 \pm 0.1$ & $6.3 \pm 0.4$ & $17.8 \pm 1.5$ \\
\hline Wrinkle free & $2.2 \pm 0.1$ & $12.7 \pm 1.0$ & $14.1 \pm 1.1$ & $2.3 \pm 0.1$ & $5.8 \pm 0.4$ & $15.8 \pm 1.3$ \\
\hline Dyed & $2.5 \pm 0.1$ & $12.8 \pm 1.0$ & $13.5 \pm 1.5$ & $2.3 \pm 0.1$ & $6.0 \pm 0.7$ & $14.7 \pm 0.9$ \\
\hline Untreated & $2.3 \pm 0.1$ & $12.0 \pm 1.0$ & $13.3 \pm 1.6$ & $2.3 \pm 0.1$ & $5.2 \pm 0.5$ & $14.4 \pm 1.0$ \\
\hline
\end{tabular}

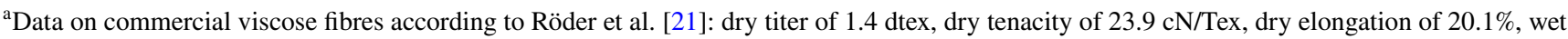
tenacity of $12.5 \mathrm{cN} / \mathrm{Tex}$, and wet elongation of $22.0 \%$

at the high end of the acceptable range, which is not preferred, whereas the $K_{r}$ value of the untreated cotton pulp indicated that it was unsuitable for the viscose process. Higher $K_{r}$ values were correlated with higher pressures in the spinneret (results not shown). Obviously, the standard viscose conditions for commercial pulps were not suitable for the new untreated cotton fabric raw material. According to Strunk et al. [20], the total weight analysis of preaged pulps before xanthation is of particular interest when introducing new pulps to the viscose process to obtain optimal viscose solution and fibre quality.

Regarding the mechanical properties of the spun filaments, the results in Table 5 indicate that there were no significant differences in dry tenacity and elongation between the treated and untreated cotton samples.

The dry tenacities were $12.0-13.8 \mathrm{cN} /$ tex, slightly lower than those of commercial viscose fibres obtained with the bench-scale equipment used here (values of $16-19 \mathrm{cN} /$ tex are normally achieved). Only the wet tenacity and wet elongation values of the combined dyed and wrinkle-free treated fabric were significantly higher than those of the untreated material. It is important to recall that the untreated reference pulp was not recommended for viscose production. Consequently, this affected the evaluation of the mechanical properties of the fibres. The accumulated pressure in the spinneret and the high $K_{r}$ values indicated the presence of undissolved gel particles, which may have negatively influenced the mechanical properties of the spun fibres. Reducing the amount of undissolved gel particles is therefore desirable to improve the filterability and spinning performance. Optimization is needed to obtain a suitable spinnable cotton reference sample before any conclusions can be drawn regarding the impact of stripped reactive dyes and DMDHEU wrinkle-free finish on the resulting mechanical properties. However, it is obvious from the filterability results that bleached reactive dyeing and wrinkle-free DMDHEU finishing of cotton fabrics positively affect the filterability and thereby the spinning performance of the produced viscose fibres. Additional characterization of the gel particles, the supramolecular properties of the cellulose polymer, and possible dye and wrinkle-free finish residues would give a better picture of the cotton substrates and of how to reduce the amount of undissolved gel particles. According to Haule et al. [22], it is the amorphous fraction of the cellulose polymer that is modified by the crosslinking agent. The amorphous part has also been shown to correlate with the accessibility of the fibres to the solvent [23].

\section{Conclusions}

This paper proposes a sequential alkaline/acid bleaching sequence to convert DMDHEU-treated wrinkle-free and reactively dyed cotton resources, through viscose regeneration processing, into cellulosic fibres. It was evident that reactive dyes and wrinkle-free finish must be removed in a pretreatment step to obtain a suitable limiting viscosity number and be able to further process the cotton resources into viscose fibres. In line with previous literature, FTIR and elemental nitrogen analysis confirmed the removal of the DMDHEU wrinkle-free agent by the alkaline/acid stripping sequence. The cotton pulps stripped of reactive dye and wrinkle-free finish could then be spun into viscose fibres. It was clearly demonstrated that the stripped cotton pulps had better filterability, i.e., a lower filter-clogging number, than did the untreated cotton pulp, which improved the viscose dope quality and spinnability of the resulting fibres. Similar mechanical properties were obtained for the stripped and untreated pulps, though the mechanical properties of the spun fibres were poorer than those of commercial viscose fibres. It is therefore recommended that the amount of gel particles in the viscose dope be reduced to enhance the spinnability and likely improve the mechanical properties. Despite that, the proposed alkaline/acid stripping approach has potential to be used to produce viscose fibres from postproduction and post-consumer cotton garment resources. Future work will focus on adapting the cotton substrate to the viscose process to produce second-lifetime viscose fibres with mechanical properties similar to those of virgin viscose fibres. 
Acknowledgements The researchers acknowledge the support of the Swedish funding agency Mistra via the programmes Future Fashion and Closing the Loop, the Swedish funding agency Vinnova via the Waste to Gold project, and the initiative Excellence in Production Research (XPRES). The authors thank MoRe Research, Sweden, for the viscose dissolution and spinning trials and technical support. The authors would further like to thank Senstex for supplying the cotton fabrics.

Author Contributions All authors contributed to the experimental planning and set-up, data analysis, and manuscript preparation. HW, EN, ZAM, and CL carried out the experimental work and data collection.

\section{Compliance with Ethical Standards}

Competing interests The authors declare that they have no competing interests.

Open Access This article is distributed under the terms of the Creative Commons Attribution 4.0 International License (http://creativeco mmons.org/licenses/by/4.0/), which permits unrestricted use, distribution, and reproduction in any medium, provided you give appropriate credit to the original author(s) and the source, provide a link to the Creative Commons license, and indicate if changes were made.

\section{References}

1. Eichinger D (2012) Lenzing Ber 90:1-7

2. Schmidt A, Watson D, Roos S, Askham C, Brunn Poulsen P (2016) Gaining benefits from discarded textile. LCA of different treatment pathways. Nordisk Ministerråd, Denmark

3. Palme A, Idström A, Nordstierna L, Brelid H (2014) Cellulose 21:4681-4691

4. Paul R (2015) Functional finishes for textiles: improving comfort, performance and protection. Woodhead Publishing in association with the Textile Institute, Cambridge
5. Cooke TF, Weigmann HD (1982) Text Chem Color 14:136-144

6. Haule LV, Carr CM, Rigout M (2014) Cellulose 21:2147-2156

7. Abhyankar PN, Beck KR, Ladisch CM, Bertoniere NR (1987) Text Res J 57:395-400

8. Haule LV, Carr CM, Rigout M (2016) J Text Inst 107:23-33

9. Zhanhong W, ZhengJun Y, Jintang Z, Yong Z (2017) Carbohydr Polym 157:945-952

10. Bigambo P, Liu H, Broadbent PJ, Carr CM, Rigout M (2018) Dyes Pigm 148:91-98

11. Abhyankar PN, Beck KR, Ladisch CM, Rowland SP (1985) Text Res J 55:444-448

12. Abhyankar PN, Beck KR, Ladisch CM, Frick JG (1986) Text Res J 56:551-555

13. Haule LV, Carr CM, Rigout M (2016) J Clean Prod 112:4445-4451

14. Lewis DM (2011) In: Clark M (ed) Handbook of textile and industrial dyeing. Woodhead Publishing, Cambridge. pp 303-364

15. Treiber E (1961) Monat Sh Chem verw Tl 93:455-468

16. Götze K (1967) Chemiefasern, Nach dem Viskoseverfahren. Springer, Berlin

17. Treiber E, Rehnström J, Ameen C, Kolos F (1962) Papier 16:85-94

18. Zellcheming (1968) Verein der Zellstoff- und Papierchemiker und -ingenieure, Merkblatt III/6B/68, Prüfung von Viskose: Filterverstopfungszahl von Viskose

19. Schleicher H, Borrmeister B (1998) Lenzing Ber 78:7-11

20. Strunk P, Lindgren Å, Eliasson B, Agnemo R (2012) Cell Chem Technol 46:556-569

21. Röder T, Moosbauer J, Kliba G, Schlader S, Zuckerstätter G, Sixta H (2009) Lenzing Ber 87:98-105

22. Haule LV, Rigout M, Carr CM, Jones CC (2012) Cellulose 19:1023-1030

23. Haule LV, Rigout M, Carr CM (2016) Carbohydr Polym 144:131-139 